\title{
Treatment of Open Book Pelvic Fractures: Comparison Between Internal and External Fixation
}

\author{
Md. Zulfiqur Ali ${ }^{1, ~ *, ~ J i b a n a n d a ~ H a l d e r ², ~ N a h i d ~ S u l t a n a ~}{ }^{3}$, Mohammad Rajib Mahmud ${ }^{4}$ \\ ${ }^{1}$ Department of Orthopaedic Surgery, Shaheed Mansur Ali Medical College, Sirajganj, Bangladesh \\ ${ }^{2}$ Department of Orthopaedic Surgery, National Institute of Traumatology \& Orthopaedic Rehabilitation (NITOR), Dhaka, Bangladesh \\ ${ }^{3}$ Department of Gynaecology \& Obstetrics, Rajshahi Medical College Hospital, Rajshahi, Bangladesh \\ ${ }^{4}$ Department of Orthopaedic Surgery, 250 Bedded Bangamatqa Sheikh Fazilatunnesa Mujib General Hospital, Sirajganj, Bangladesh
}

\author{
Email address: \\ dr.shaju123@gmail.com (Md. Z. Ali) \\ ${ }^{*}$ Corresponding author
}

\section{To cite this article:}

Md. Zulfiqur Ali, Jibananda Halder, Nahid Sultana, Mohammad Rajib Mahmud. Treatment of Open Book Pelvic Fractures: Comparison Between Internal and External Fixation. Journal of Surgery. Vol. 9, No. 3, 2021, pp. 145-148. doi: 10.11648/j.js.20210903.18

Received: March 1, 2021; Accepted: April 21, 2021; Published: June 4, 2021

\begin{abstract}
Background: An open-book pelvic fracture is used to describe any fracture that significantly disrupts the pelvic ring of human body. Internal and external fixation in treating open book pelvic fractures are two broadly used treatment methods. But we have very few comparative data regarding this issue. The aim of this study was to compare both internal and external fixation methods in the treatment of open book pelvic fractures. Methods: This prospective comparative study was conducted at the Department of Orthopaedic Surgery in Shaheed Mansur Ali Medical College, Sirajganj, Bangladesh and The National Institute of Traumatology \& Orthopaedic Rehabilitation (NITOR) during the period from January 2016 to December 2018. In total 100 patients with open-book pelvic fracture were obeyed all the inclusion and exclusion criteria and completed the full tenure of the intervention were finalized as the study population. Total population were divided into two groups. In Group I there were 50 participants who were treated by internal fixation method and in Group II there were 50 participants who were treated by external fixation method. Data were analyzed by SPSS Version 20 and disseminated by MS Office programs. Result: In the final follow-up of this study among total participants, there were 34 excellent, 30 good, 16 fair and 20 poor results. But, In Group I, 56\% got excellent and 38\% got good result. Besides these, only $4 \%$ got fair and $2 \%$ got poor result. On the other hand, the highest $38 \%$ participants got poor result. Then $28 \%, 22 \%$ and $12 \%$ got fair, good and excellent results respectively. This difference was statistically highly significant, the p-value was 0.013 . Conclusion: After completing this comparative study it was found that the internal regarding outcome and complications the internal fixation method is better than external fixation method in treating open-book pelvic fracture.
\end{abstract}

Keywords: Open Book, Pelvic Fractures, Internal Fixator, External Fixator

\section{Introduction}

Every fracture that suggestively interrupts the pelvic ring of human body is defined by an open-book pelvic fracture. External and internal fixation are two largely used treatment procedures in the management of open book pelvic fractures. An extensive amount of consideration has been given to pelvic fractures in the Orthopaedic literature as the early explanation of a double vertical fracture as a mixture of pubic rami fractures and a fracture of the iliac wing by French surgeon Joseph Malgaigne in his 1859 atlas of traumatology, having to do with their complication and related morbidity and mortality [1]. It is reported that, $2 \%$ to $8 \%$ of all skeletal injuries are fractures of the pelvic ring [2]. And are frequently linked with high-energy shock, motor vehicle accidents and falls from a height mostly. Prevalence of pelvic fracture seems to be cumulative, inferior to increases in the number of high-speed motor vehicle accidents and the number of patients surviving these accidents, because of airbags and harmless car designs. Nearly $20 \%$ have pelvic damages in the midst of increase injured patients with blunt trauma. Evidence showed that, the pelvic fractures represent 
$3 \%$ of all fractures [3]. Stable and unstable, are the two types of fractures seen in adult pelvis. Stable fractures come from low energy trauma for example, falls in old patients and are treated symptomatically with supports or walker help out ambulation. Conversely, unstable fractures are triggered by high energy trauma and bring about noteworthy morbidity as well as mortality. Similar to other bones, pelvis is likewise visible to trauma. And the rate of the pelvic fracture is increasing gradually. These kinds of fractures are associated with an increasing mortality rate, which is approximately $25 \%$. The leading cause of death is retroperitoneal hemorrhage [4]. A study revealed that, around 5\% patients of pelvic fractures involve hospital admission, amongst them 5 to $16 \%$ die regardless of treatment [5]. Categorizing the pelvic fractures, Tile considered the pelvic fracture into three types (A, B \& C). The commonness is $16 \%$ of Type " $\mathrm{A}$ " stable pelvic fracture, $49 \%$ of Type " $\mathrm{B}$ " rotationally unstable pelvic fracture and Type " $\mathrm{C}$ " vertically and rotationally unstable is $35 \%$. [6] Basically, two methods are applied in the treatment of this type of unstable pelvic fractures. These are: i) close reduction \& external fixation, and ii) open reduction and internal fixation. [7] Internal fixation, external fixation is applied in an inclusive series in open-book pelvic fracture treatment. The stabilization of pelvic fractures via external fixation has shaped a transformation in the treatment of poly shock sufferers, although numerous modalities of stabilization of pelvic fractures have been presented. External fixation acts not only as a role of resuscitative measure varying intensely but also the survival percentage [8]. Besides, the provisional part, external fixation similarly meet the expense of absolute management, even though, the stabilization of pelvic fractures decreases the hemorrhage inside the pelvic ring.

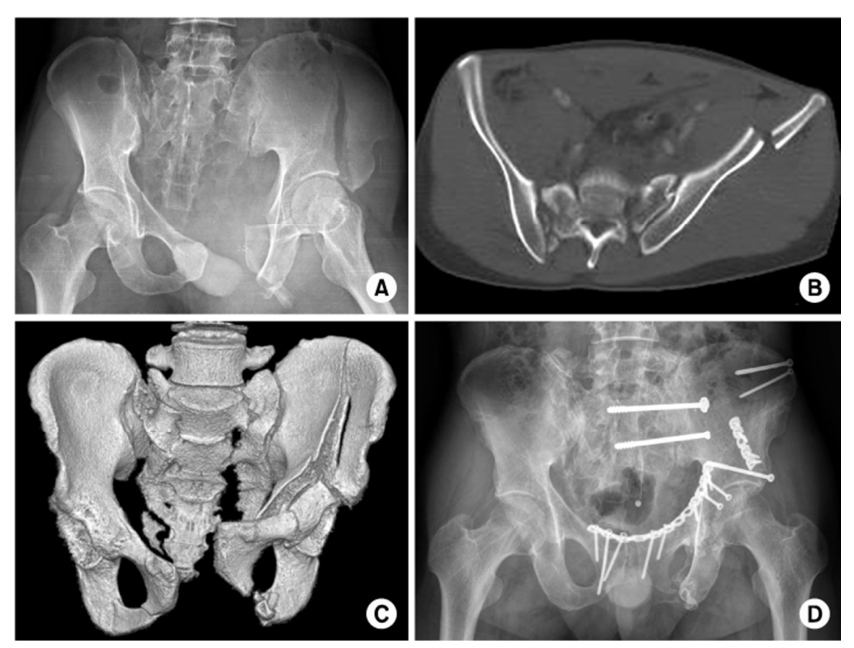

Figure 1. Radiology of Pelvic Fracture.

\section{Objectives}

General Objective:

To compare internal and external fixation methods in open-book pelvic fracture.

Specific Objective:
To find out the complications regarding both the fixation methods.

\section{Methodology \& Materials}

This prospective comparative study was conducted at the Department of Orthopaedic Surgery in Shaheed Mansur Ali Medical College, Sirajganj, Bangladesh and The National Institute of Traumatology \& Orthopaedic Rehabilitation (NITOR) during the period from January 2016 to December 2018. One hundred (100) patients with open-book pelvic fracture were obeyed all the inclusion and exclusion criteria and completed the full tenure of the intervention were finalized as the study population. Total population were divided into two groups. In Group I there were 50 participants who were treated by internal fixation method and in Group II there were 50 participants who were treated by external fixation method. Patients of both sex age $>15$ years come within 1 week of injury were involved in our study. Patients who were excluded: i) $<15$ years and $>50$ years old, ii) open pelvic fractures, iii) Tile's type $A$ and $C$, iv) patients with metabolic bone disease and v) rheumatoid and other inflammatory arthritis. All patients were assessed for skeletal and visceral damages by x-rays, USG \& CT scan after primary fluid resuscitation. Painkillers, $\mathrm{i} / \mathrm{v}$ antibiotics and prophylaxis counter to D. V. T were delivered. External pelvic support in the form of pelvic binders were provided to all the patients and organized for final surgical system. Internal fixation was applied in Group I for pelvic stabilization over a low crosswise cut. Cutting up was performed to the symphyseal separation and sub-periosteally the larger and therapeutic characteristics of the pubic bones were visible. In Group II, once aseptic trials Schanz screws were injected on both side. All screws were detained by a rod and exterior compression device. Patients were organized with limited weight bearing using walker and discharged on 3rd post-op day. Time frame and assessment tools were equivalent in both the groups. All patient was discharged on 3-4 post-operative days afterward the surgery. Moreover, every patient was recommended to follow up at two weeks for elimination of suture, next follow up each four weeks for 24 weeks. The functional outcome was assessed at each follow up visit via Majeed pelvic scores, clinically \& radiologically. According to this scoring, for pain, work, sitting, sexual intercourse and standing status 30, 20, 10, 4 and 36 points were assigned respectively. Total point was 100 . Besides these in final outcome $>85,70-84,55-69$ and $<55$ points were the indicators of excellent, good, fair and poor performance of the treatment. Data were analyzed by SPSS Version 20 and disseminated by MS Office programs.

\section{Result}

In this study, the mean age of patients was $37.38 \pm 8.56$ with range 15-60 years. Among total participants $72 \%$ were male and $28 \%$ were female. So male were dominating in number here. The pelvic fracture was dependent of gender 
that was predominantly in males, $p$-value $<0.006$. In this study $\mathrm{p}$ value of less or equal of 0.05 was considered as significant correlation. In the final follow-up of this study among total participants, there were 34 excellent, 30 good, 16 fair and 20 poor results. But, In Group I, 56\% got excellent and $38 \%$ got good result. Besides these, only $4 \%$ got fair and $2 \%$ got poor result. On the other hand, the highest $38 \%$ participants got poor result. Then $28 \%, 22 \%$ and $12 \%$ got fair, good and excellent results respectively. This difference was statistically highly significant, the p-value was 0.013 . The injuries of bowel system were suspected in $25 \%$ cases. The general surgeon was consulted and were treated conservatively. Pin tract infection rate was $10 \%$ in Group I and $22 \%$ in Group II. The superficial wound infection was found $8 \%$ in Group I and $18 \%$ in Group II. In Group I there were deep infection in only $3(6 \%)$ and breakage of Schanz screw were in $2(4 \%)$ cases. But in Group II deep infection were found in $7(14 \%)$ and breakage of Schanz screw were found $6(12 \%)$ cases.

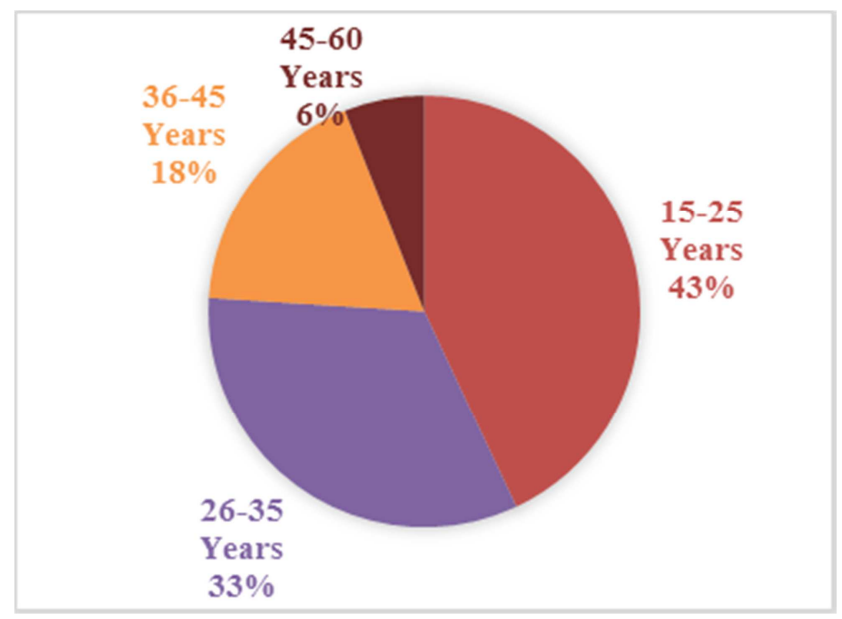

Figure 2. Age distribution of participants $(N=100)$.

Table 1. Result of the two groups at final follow-up $(N=100)$.

\begin{tabular}{lllllll}
\hline \multirow{2}{*}{ Category } & Group I & & Group II & & Total \\
\cline { 2 - 6 } & $\mathbf{n}$ & $\mathbf{\%}$ & $\mathbf{n}$ & $\mathbf{\%}$ & $\mathbf{N}$ \\
\hline Excellent & 28 & $56 \%$ & 6 & $12 \%$ & 34 \\
Good & 19 & $38 \%$ & 11 & $22 \%$ & $34 \%$ \\
Fair & 2 & $4 \%$ & 14 & $28 \%$ & $30 \%$ \\
Poor & 1 & $2 \%$ & 19 & $38 \%$ & 16 & 20 \\
\hline
\end{tabular}

Table 2. Complication among participants of both groups $(N=100)$.

\begin{tabular}{lllll}
\hline \multirow{2}{*}{ Complications } & Group I & & Group II \\
\cline { 2 - 5 } & $\mathbf{n}$ & $\mathbf{\%}$ & $\mathbf{n}$ & \\
\hline Pin tract infection & 5 & $10 \%$ & 11 & \\
Superficial wound infection & 4 & $8 \%$ & 9 & $22 \%$ \\
Deep infection & 3 & $6 \%$ & 7 & $18 \%$ \\
Breakage of Schanz screw & 2 & $4 \%$ & 6 & $14 \%$ \\
\hline
\end{tabular}

\section{Discussion}

The aim of this study was to compare both internal and external fixation methods in the treatment of open book pelvic fractures. The unstable pelvic disruptions are life threatening and their stabilization is one of the priorities. [9] In total 100 patients with open-book pelvic fracture were obeyed all the inclusion and exclusion criteria and completed the full tenure of the intervention were finalized as the study population. Total population were divided into two groups. In Group I there were 50 participants who were treated by internal fixation method and in Group II there were 50 participants who were treated by external fixation method. Unstable pelvic fracture was treated by Mears 1980, by external fixator. 30 patients were excluded at mean follow up (over 5-6 years), as main acetabular participation sendoff 218 cases and $47.5 \%$ cases exposed inacceptable results owing to several difficulties similar to leg length discrepancy (4\%), non-union (35\%) and pain (40\%) [10]. External fixation has been broadly defined for the final treatment of type B-2 fractures. Reduction in $83 \%$ for type B-2 injuries was obtained and maintained by Kellam (1989), through the practice of external fixator and determined that if a satisfactory decrease $(<1 \mathrm{~cm}$ displacement) was continued, $100 \%$ of patients were functionally ordinary [11]. In a different study, Cole, et al (1996) described the maximum of the difficulties related to external fixation are connected with a failure to accomplish satisfactory stabilization of the posterior part of the pelvic ring. As result, there have been reports of posterior pelvic pain [12]. In a study it was observed that, 110 patients with unbalanced fractures that had been treated by external fixator that the percentage of difficulties was high; by loss of reduction $57 \%$. Mal-union (58\%), non-union (5\%), pin track infection (24\%) and loosing of pins was $(2 \%)$ evaluated in their study [13]. In Group I pin tract infection rate was $10 \%$ and the superficial wound infection was $8 \%$. Meanwhile, in Group II $22 \%$ pin tract infection rate and $18 \%$ superficial wound infection was observed. Also, deep infection in only $3(6 \%)$ and breakage of Schanz screw were in $2(4 \%)$ cases in Group I. But then again, in Group II deep infection were found in 7 (14\%) and breakage of Schanz screw were found $6(12 \%)$. In a study of Tornetta et al with 29 patients, Follow-up assessment exposed that $96 \%$ had no pain otherwise pain simply with strenuous activity. $76 \%$ ambulated without support or 
restrictions, plus $76 \%$ returned to their pre-injury profession [14]. Awais and Rizwan 1996 treated 10 cases unbalanced pelvic fracture through tension band wiring. They stated that this method delivers steady fixation in unstable pelvic fracture [15]. In the final follow-up of our study among total participants, there were 34 excellent, 30 good, 16 fair and 20 poor results. But, In Group I, 56\% got excellent and 38\% got good result. Besides these, only $4 \%$ got fair and $2 \%$ got poor result. On the other hand, the highest 38\% participants got poor result. Then $28 \%, 22 \%$ and $12 \%$ got fair, good and excellent results respectively. In the study of Maru, he testified with Majeed Pelvic score and observed that, there were 9 excellent, 8 good, 1 fair and 1 poor results so along with outcomes internal fixation open reduction \& internal fixation with plate provides the recovering outcomes [16].

Limitations of the study:

This was a single centered study with a small sized sample. So the findings of this study may not reflect the exact scenario of the whole country.

\section{Conclusion}

After completing this comparative study it was found that regarding outcome and complications the internal fixation method is better than external fixation method in treating open-book pelvic fracture.

\section{Recommendations}

For getting more specific findings we would like to recommend for conducting more studies regarding the same issue with larger sample size.

\section{References}

[1] Malgaigne JF. Double vertical fractures of the pelvis. 1859. Clin Orthop Relat Res. 2007; 458: 17-9.

[2] Grotz MR, Allami MK, Harwood P, et al. Open pelvic fractures: epidemiology, current concepts of management and outcome. Injury. 2005; 36 (1): 1-13.
[3] Bailey \& Love: Short practice of surgery. 24th Edition international Edition Arnold publishers London 2004: 387-388.

[4] Rittmeister M, Lindsey RW, Kohl HW: Pelvic fracture among polytrauma decedents. Trauma based mortality with pelvic fracture a case series of 74 patients. $3^{\text {rd }}$. The ben Taub general hospital, Baylor College of medicine, Houston, U S A. Arch Ortho trauma surg. 2001; 121 (1): 43-9.

[5] Pereira SJ, O'Brien DP, Luchette FA, et al: Dynamic helical computed tomography scan accurately detects hemorrhage in patients with pelvic fracture. Surgery 2000; 128 (4): 678-85.

[6] Tile M. Pelvic ring fractures: should they be fixed. J Bone Joint SurgBr 1988; 70: 1-12.

[7] Tile M. Pelvic fractures: operative versus non-operative treatment. OrthopClin North Am. 1980; 11: 423-464.

[8] Kellam JF. The role of external skeletal fixation in pelvic disruptions. Clinical Orthopedics' and Related Research. 1989; 241: 66-80.

[9] Craveiro LN, Escalda C, Tavares D, et al. Treatment of pelvic ring disruptions with the Ilizarov frame. Preliminary report. Journal of Bone and joint surgery - British volume 2006; 88-B: 160.

[10] Riska EB, Bonsdroff HV, Hakkinen S, et al: External fixation of unstable pelvic fractures. IntOrthop 1979; 3: 183-188.

[11] Kellam JF: The role of external fixation in pelvic disruption. Clin. Orth. 1989; 241: 66-82.

[12] Cole JD, Blum DA, Ansel LJ. Outcome after fixation of unstable posterior pelvic ring injuries. ClinOrthop. Aug 1996; (329): 160-79.

[13] Lindahl J, Hirvensalo E, Bostman O, Santavirta S. Failure of reduction with an external fixator in the management of injuries of the pelvic ring. Long-term evaluation of 110 patients. J Bone Joint Surg Br. 1999; 81: 955-962.

[14] Tornetta P, III, Dickson K, Matta JM. Outcome of rotationally unstable pelvic ring injuries treated operatively. ClinOrthop. 1996; $147-151$

[15] Awais SM, Rizwan N: The Journal of Pakistan Orthopaedic Association 1996; 1 (12).

[16] Maru M. The functional outcome of surgically treated unstable pelvic fractures. J. Orthopaedics 2005; 2 (2): 2 . 